\title{
SIMULATION AND ANALYSIS OF EXPRESS RUN FREQUENCY
}

\author{
David Williams \\ J.O. Miller \\ Dan Mattioda \\ Department of Operational Sciences \\ Graduate School of Engineering and Management \\ Air Force Institute of Technology \\ Wright-Patterson AFB, OH 45433, USA
}

\begin{abstract}
EXPRESS is a database tool the Air Force (AF) uses to prioritize depot maintenance of reparable spare parts based on warfighter need. Many studies have examined individual portions of EXPRESS, though few examine it as an entire system. This effort proposes a modeling approach for examining overall system behavior of EXPRESS using discrete event simulation. The emphasis of the model is to be flexible enough to provide useful insight into system performance and a foundation for future expansion and analysis. In current operations, EXPRESS runs every day. This research effort tries to determine the impact on the depot repair process' ability to respond to warfighter need due to running EXPRESS less frequently. A discrete event simulation written in Arena, modeling the general flow of information and parts through the depot repair process, is used to determine the effect of the frequency of EXPRESS runs on Mission Capable (MICAP) hours.
\end{abstract}

\section{INTRODUCTION}

Prioritizing depot maintenance is a very important issue for the AF. Nearly every weapon system in the inventory relies on depot maintenance to stay mission capable. Reparable parts that fail which cannot be fixed at their operating base are sent for repair to one of the Air Force Material Command (AFMC) Air Logistics Centers (ALCs). The constraints imposed at an ALC by carcass availability, repair resource capacity, budget, and replacement parts require that maintenance be prioritized in order to most effectively address warfighter needs. This is accomplished by the Execution and Prioritization of Repair Support System (EXPRESS): a database tool managed by the Air Force Sustainment Center (AFSC) that takes into account real time position data from the depot supply chain to produce a prioritized list of maintenance and distribution actions that maximize the likelihood that the fleet's availability goals are met for the least cost.

In current operations, EXPRESS runs every day. This research effort tries to determine the impact on the depot repair process' ability to respond to warfighter need due to running EXPRESS less frequently: something highly desirable to maintenance planners attempting to maximize efficiency and load leveling at an ALC. A discrete event simulation written in Arena, modeling the general flow of information and parts through the depot repair process, is used to determine the effect of the frequency of EXPRESS runs on MICAP hours.

This paper begins with a brief background reviewing some of the history of EXPRESS. We then describe our strategy for modeling the EXPRESS process and details of the scenario used for our simulation study. Results and analysis are presented along with suggestions for future research. 
Williams, Miller, Mattioda

\section{BACKGROUND}

EXPRESS is a combination of several supply chain management tools that were merged into a single hierarchy in the 1990s (Air Force Material Command 1995 and 2004). It employs a prioritization algorithm derived from the Distribution and Repair in Variable Environments (DRIVE) model developed by AFMC and the RAND Corporation in the 1980s (Crawford 1988; Abell et al. 1992; Cohen et al. 1991; Miller et al. 1992). Its objective function is to maximize the likelihood that aircraft availability goals, based on warfighter scenarios over short planning horizons, are achieved given the highly variable nature of part failures (Moore and McCormick 1992). EXPRESS also uses logic from the supportability module developed at the Ogden ALC to "examine whether or not the items needing repair were supportable for repair parts and other resources" (Air Force Material Command 1995). Additionally, several tasks previously requiring manual input were automated within EXPRESS by incorporating logic from the Automated Induction System (AIS) developed at the Oklahoma City ALC (Air Force Material Command 2004). Together these functions allow EXPRESS to serve as the single AFMC process for determining which items to put into repair.

The motivation for EXPRESS comes out of the Depot Repair Enhancement Process (DREP) which focused on streamlining repair processes and more closely aligning them with warfighter needs (Air Force Material Command 2001). One of the key measurements of logistics performance highlighted by DREP is MICAP hours. Another performance indicator, Customer Wait Time (CWT), is a measure of total wait time for a customer from the time they submit a need until it is fulfilled (Department of Defense 2000). MICAP hours is a special subset of CWT reserved for requirements that represent a mission capability need (i.e. an aircraft is grounded until the requirement is fulfilled). MICAP hours is the primary measure of system performance studied by this effort.

The AF has often questioned what the impact would be if EXPRESS were run less often (Air Force Material Command 2012). Several practical studies have been executed at different ALCs with a subset of shops running EXPRESS weekly instead of daily (Air Force Material Command 2011). No rigorous analytical output was produced during these studies, and subject matter experts could not determine that the resulting increase in shop efficiency outweighed the reduction in responsiveness to customer demand. AFMC has previously studied this question using a computer simulation, with the results pointing to potential gains in aircraft availability due to less frequent runs (Stafford 2002). Inconclusive evidence, along with continued debate between the two EXPRESS-user communities of depot maintenance and supply chain managers, leaves the periodicity of EXPRESS runs a point of debate. This effort attempts to shed light on the debate by revisiting the problem with a computer simulation.

\section{METHODOLOGY}

\subsection{Modeling Strategy}

A discrete event simulation was developed to model the flow of requirements through EXPRESS, and the resulting parts that are maintained and distributed. In order to limit the scope of this study to a manageable size, the boundaries of the model are limited to the walls of a notional ALC. Within these walls two repair shops are modeled, along with a subset of the parts they repair. The shops were selected based on subject matter expert opinion of examples representing the spectrum of supportability constraint behavior. Thus the first notional shop represents one repairing small parts for the F-16. These parts require only a few hours of labor to complete and repairs demand little from the ALC budget. The other represents a structures shop repairing large parts for the $\mathrm{KC}-135$, each requiring many hours of labor and many days to fix.

Of the parts repaired by these shops, only three are modeled: two from the small parts shop and one from the structures shop. Data was collected from archived EXPRESS tables from 3 January 2011 to 30 June 2011. Queries on average rank value and portion of shop labor were used to map simulation parame- 
ters to real world parts. The three modeled parts represent National Item Identification Numbers (NIIN) 13130343 (F-16 assembly), 13903690 (F-16 assembly), and 1095725 (KC-135 refueling boom). Table 1 summarizes the parameters found during data collection over the modeled period for each part.

Table 1: Part parameter summary.

\begin{tabular}{ccccccc}
\hline NIIN & $\begin{array}{c}\text { Model } \\
\text { Part No. }\end{array}$ & $\begin{array}{c}\text { Working } \\
\text { Level }\end{array}$ & $\begin{array}{c}\text { Failure } \\
\text { Rate }\end{array}$ & $\begin{array}{c}\text { Workload } \\
\text { Portion }\end{array}$ & $\begin{array}{c}\text { Planning } \\
\text { Horizon } \\
\text { (Days) }\end{array}$ & $\begin{array}{c}\text { Maintenance Time } \\
\text { Distribution } \\
\text { (Days) }\end{array}$ \\
\hline 13130343 & 1 & 13 & 0.123 & $89 \%$ & 74 & $1+$ EXPO(73.4) \\
13903690 & 2 & 7 & 0.071 & $29 \%$ & 92 & $1+$ EXPO(91.1) \\
1095725 & 3 & 18 & 0.292 & $18 \%$ & 94 & $1+$ \\
& \\
\hline
\end{tabular}

The number of individual bases operating F-16s and KC-135s, and therefore using the three modeled parts, number in the hundreds. Instead of individually modeling each base, demand and priority behavior were aggregated across the fleet and broken into three notional user bases, with each base designated by a separate Stock Record Account Number (SRAN). Table 2 outlines these notional bases and their demand rates.

Table 2: Requirement generation parameters by SRAN and NIIN.

\begin{tabular}{cccccc}
\hline $\begin{array}{c}\text { Notional } \\
\text { SRAN }\end{array}$ & NIIN & Model Part No. & $\begin{array}{c}\text { Aggregated } \\
\text { Rate }\end{array}$ & $\begin{array}{c}\text { \% Fleet Mod- } \\
\text { eled }\end{array}$ & Modeled Rate \\
\hline 1 & 13130343 & 1 & 0.0708 & 30 & 0.021 \\
1 & 13903690 & 2 & 0.1233 & 30 & 0.037 \\
2 & 13130343 & 1 & 0.0708 & 70 & 0.050 \\
2 & 13903690 & 2 & 0.1233 & 70 & 0.086 \\
3 & 1095725 & 3 & 0.2920 & 100 & 0.292 \\
\hline
\end{tabular}

Entities in the simulation model three types of requirement. The first represent requisitions from the field generated by actual part failures, or Back Orders (BOs). BOs are assumed to arrive according to a Poisson distribution with average daily rate equal to the notional base demand rate. Once all $\mathrm{BO}$ entities have been generated on a given day, the difference between their count and the inverse Poisson cumulative distribution function (with average rate equal to the part's average daily failure rate multiplied by the average repair time for that part) evaluated at .95 , becomes the count for the number of Prioritization of Aircraft Reparable Spares (PARS) entities generated. These entities mimic the requirements generated by the primary EXPRESS prioritization algorithm, PARS, that attempts to generate and rank repair requirements above and beyond BOs from the field in order to maximize aircraft availability across the fleet (Moore and McCormick 1992). The third type of entity models additional requirements generated in EXPRESS to ensure enough requirements are in the repair pipeline to keep adequate safety stocks at the depot. These Working Level (WL) target requirements are called WL entities.

Since the supply chain between each base and the depot are not modeled, the prioritization logic used by EXPRESS is approximated by two random number draws. The first, coming from an exponential distribution, is reserved only for those BOs with MICAP status (occurring when the notional base currently has more unmet BOs than its allowable holes value). This number emulates the Spares Priority Release Sequence (SPRS) categorization of MICAP parts. Each notional base has a different average value for the random number draw, representative of higher priority given to different bases due to their mission. The 
second is a uniform random number draw between zero and one. This value is assigned to every entity (added to the SPRS number for those that have one) and represents final fine tuning rank given by EXPRESS to each requirement.

Several assumptions were made when modeling supportability logic. The only constraint explicitly modeled is shop capacity measured in labor hours available. Each Production Shop Scheduling Designator (PSSD) has a fixed repair hour capacity which is decremented by the number of hours required to repair a part when it is inducted. Thus a part will pass the capacity constraint if there are enough remaining hours in the PSSD capacity to induct it. Historically $45-48 \%$ of requirements meeting supportability pass for carcass. Carcasses are Line Replaceable Units (LRUs) that failed previously at a base and have been shipped back to the depot for repair. Both the carcass and parts constraints represent complex supply chains that could be modeled in depth in future studies. Historically, those requirements passing for carcass pass for capacity $50 \%$ of the time. The fund constraint has largely not been a binding one in the past, though research into this aspect of the problem is of interest (Walker 2012). In the past roughly 99\% pass for funds, but for this effort $100 \%$ pass during the supportability check. Finally, of those passing for carcass, capacity, and funds, roughly $30 \%$ pass for parts. In order to roughly mimic the stochastic nature behind the carcass and parts constraints, the number of a given National Stock Number (NSN) allowed to pass each supportability constraint is calculated by

$$
\text { Allowed }_{N S N, t}=\text { Count }_{N S N, t} \times X,
$$

where $X \sim$ Exponential with mean of 0.45 for carcass, and $(.45)(.5)(1)(.3)=.07$ for parts. Requirements meeting supportability are processed in priority order one at a time. Those requirements passing all supportability constraints move on to maintenance.

Repair is modeled by a simple delay for each part based on fitted distributions of historic shop flow days data. Table 1 outlined these distributions for each part, and Table 3 shows the cost of inducting each part to the shops capacity and budget. The number of parts currently being repaired is reported by the On Work Order (OWO) variable. Once repair is complete, the number of parts OWO is decreased and the number of parts on hand is increased. Parts on hand are shipped to fulfill the highest priority need waiting to be met at the notional bases. The overall flow of the simulation logic is shown in Figure 1.

Table 3: Modeled part costs.

\begin{tabular}{cccc}
\hline NIIN & Average Rank & Average Cost (Hours) & Average Cost (\$) \\
\hline 1095725 & 101151 & 773 & $\$ 172,639$ \\
13130343 & 47695 & 21 & $\$ 16,760$ \\
13903690 & 54268 & 21 & $\$ 18,453$ \\
\hline
\end{tabular}

The modeled system performance is tracked by total CWT and MICAP days, which are convertible to hours by multiplying by 24 . CWT is tracked by measuring the total time between when a requirement is generated by a notional base, and when it is matched by the distribution process in EXPRESS. The delay time of those parts that have MICAP status are tracked by a second variable. These totals are recorded after each run, and serve as the primary measure of the system's ability to address user need. Additionally, daily counts of parts OWO, along with a myriad of system variables, are recorded for use in analysis and diagnostics.

\subsection{Periodicity}

The run frequency of EXPRESS is controlled by setting a variable that determines how many days elapse between runs. BO generation happens every day regardless of whether EXPRESS runs, while PARS enti- 
ties are generated only as a part of prioritization when EXPRESS runs. The primary assumptions regarding system behavior as a function of run frequency include:

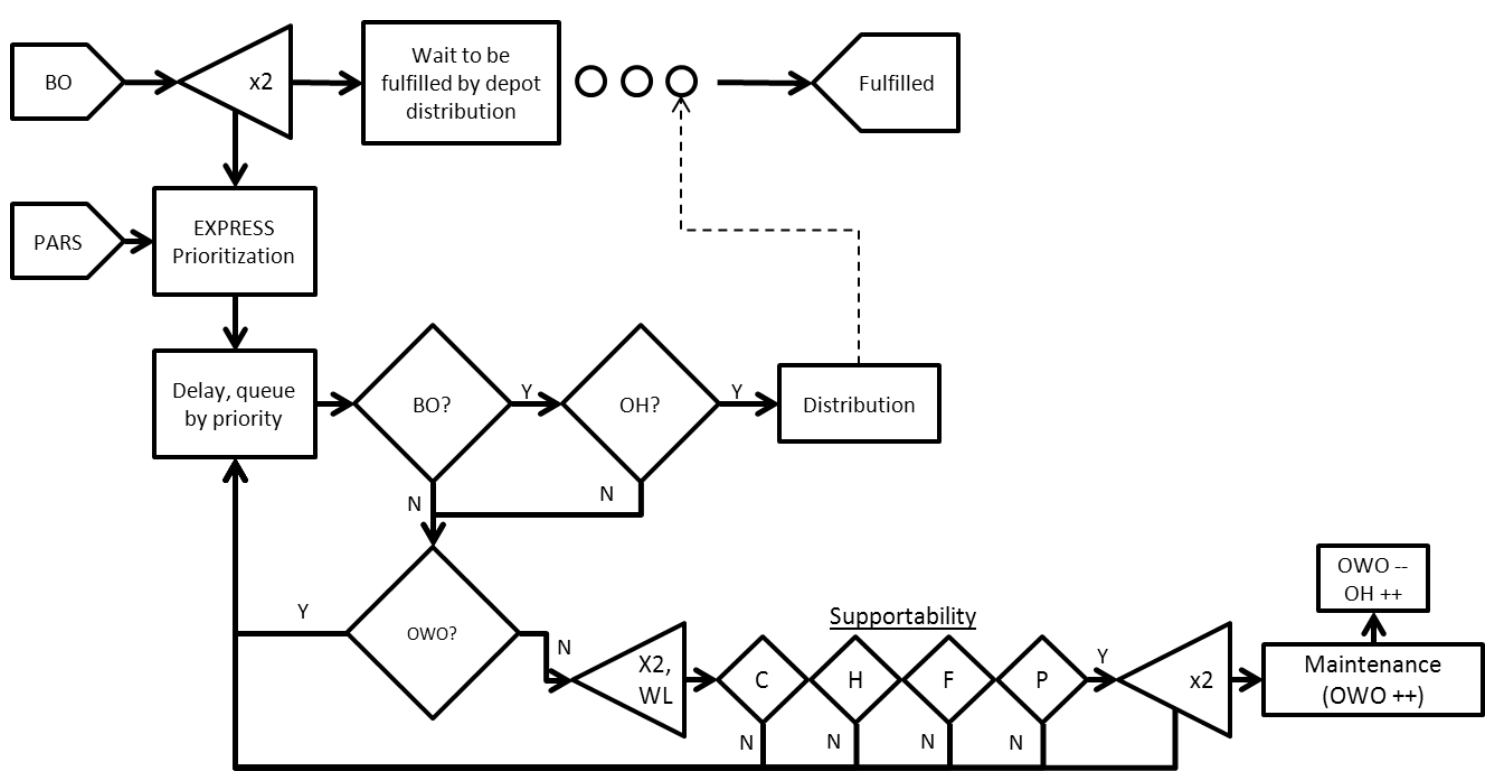

Figure 1: Overall model logic flow.

- EXPRESS runs in its entirety according to the frequency variable, and only then. Distribution is included in this, and requirements are only matched during runs.

- Notional SRAN behavior does not change with run frequency.

- Prioritization logic does not change.

- Workshop capacity limits used in supportability logic are multiplied by the number of days between runs.

- The average portion of requirements meeting supportability that pass for either carcass or parts does not change.

It is also assumed the depot repair process is, in general, a steady state system. A warm-up period of 400 days was used to reduce initialization bias prior to collecting performance metrics. This was determined by plotting system behavior over several runs and observing when performance appeared to have roughly leveled out. Since the input parameters were taken from a 6 month period of time, output data was only measured for 6 months (130 days) after the warm up period in order to avoid extrapolating outside observed system behavior. Subject matter experts on the logic flow of EXPRESS verified the model's layout and assumptions prior to implementation. The simulation was then validated against real world data for OWO daily positions and total MICAP hours while in its daily run configuration (Williams 2012).

\section{RESULTS}

The primary investigation point of this effort is to shed light on the potential impacts of running EXPRESS less frequently. The simulation was configured to run every 1, 2, 5 (weekly), 10 (every other week), and 20 days (monthly), and output data was gathered for each. Fifty runs for each system configuration were executed. Since the most important performance factor is responsiveness to customer need, total MICAP days is the first topic of analysis. The sum of total wait time for MICAP requirements was 
recorded for each individual part, as well as the collective sum for all three. Figure 2 shows the distributions of the collective sum for the different configurations.

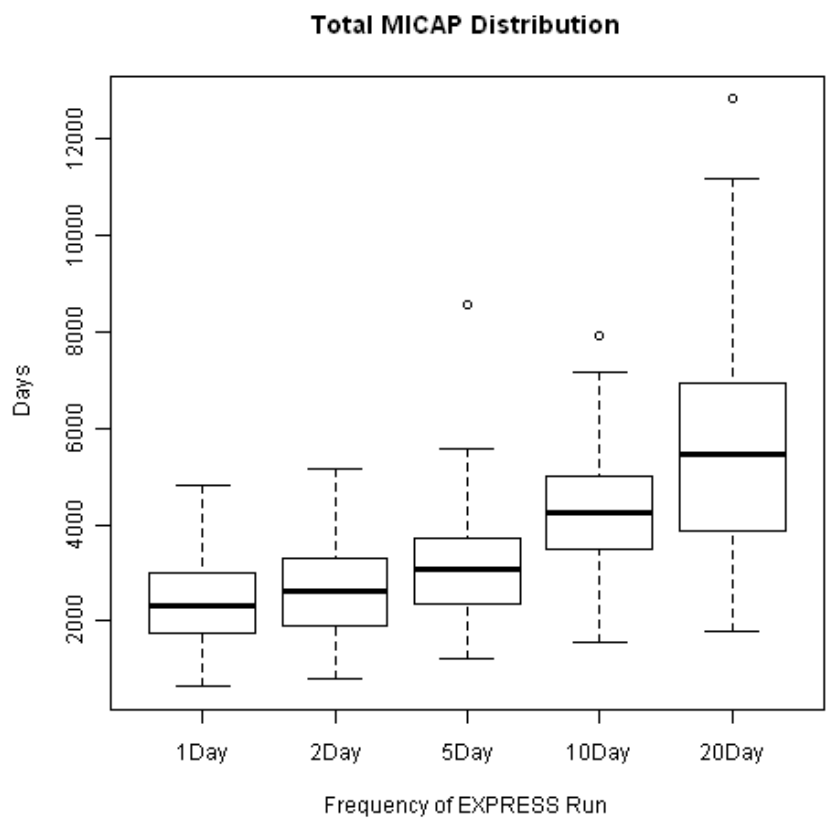

Figure 2: Distribution of total MICAP days.

A Wilcoxon Signed Rank Test is used to non-parametrically compare the results of the different configurations. In an attempt to isolate the variance between configurations for comparison to just the variance attributed to run frequency, the simulation was configured using common random numbers. Thus the paired comparison of the Wilcoxon test was used to determine the treatment effect of running EXPRESS less frequently. The results are outlined in Table 4. Since the plots in Figure 2 indicate increasing the number of days between EXPRESS runs increases the median, each p-value comes from the one-sided test checking to see if the output from the higher frequency (Frequency 1) is less than the lower frequency (Frequency 2).

Both the generally increasing quantile plots from Figure 2 and the low p-values in Table 4 (all less than .05) of the pairwise comparisons, indicate that decreases in EXPRESS frequency result in statistically higher MICAP times. The Wilcoxon Signed Rank Test does not reveal the size of the difference, just whether there is statistical evidence of one.

Table 4: Comparison of median MICAP days by run frequency: Wilcoxon signed ranked test.

\begin{tabular}{cccccc}
\hline & & \multicolumn{4}{c}{ p-values } \\
\cline { 3 - 5 } Frequency 1 & Frequency 2 & Part 1 & Part 2 & Part 3 & Total \\
\hline 1 & 2 & 0.000001 & 0.009546 & 0.000533 & 0.000005 \\
2 & 5 & 0.000000 & 0.000000 & 0.000063 & 0.000000 \\
5 & 10 & 0.000025 & 0.000000 & 0.000009 & 0.000000 \\
10 & 20 & 0.009495 & 0.013150 & 0.006916 & 0.000284 \\
\hline
\end{tabular}


There appears to be only a small shift between the 1,2 , and 5 day outputs in terms of the median response, with a much larger shift at the 10 and 20 day configurations. Additionally, the variance appears to increase with the number of days between runs as outlined in Table 5. This would be expected due to a reduction in the frequency of times workshop labor distribution can be adjusted to match changes in demand. These patterns appear to hold across the quantile plots of individual parts' MICAP times seen in Figures 3 - 5. These outputs are from a mathematical abstraction of the problem and offer only evidence of a change in behavior. The decision of how much of a shift in output distribution and increase in variance is acceptable remains the task of the EXPRESS user community.

Table 5: Summary statistics on total MICAP days by configuration.

\begin{tabular}{cccccc}
\hline $\begin{array}{c}\text { Frequency } \\
\text { (days) }\end{array}$ & Median & Mean & $\begin{array}{c}\text { Standard Devia- } \\
\text { tion }\end{array}$ & Maximum & Range \\
\hline 1 & 1180 & 1374.70 & 712.33 & 3341 & 2950 \\
2 & 1440 & 1678.34 & 802.76 & 3720 & 3118 \\
5 & 2416 & 2537.38 & 1096.21 & 5194 & 4475 \\
10 & 3752 & 3761.92 & 1226.93 & 6768 & 5697 \\
20 & 4362 & 4838.02 & 2063.25 & 10176 & 9043 \\
\hline
\end{tabular}

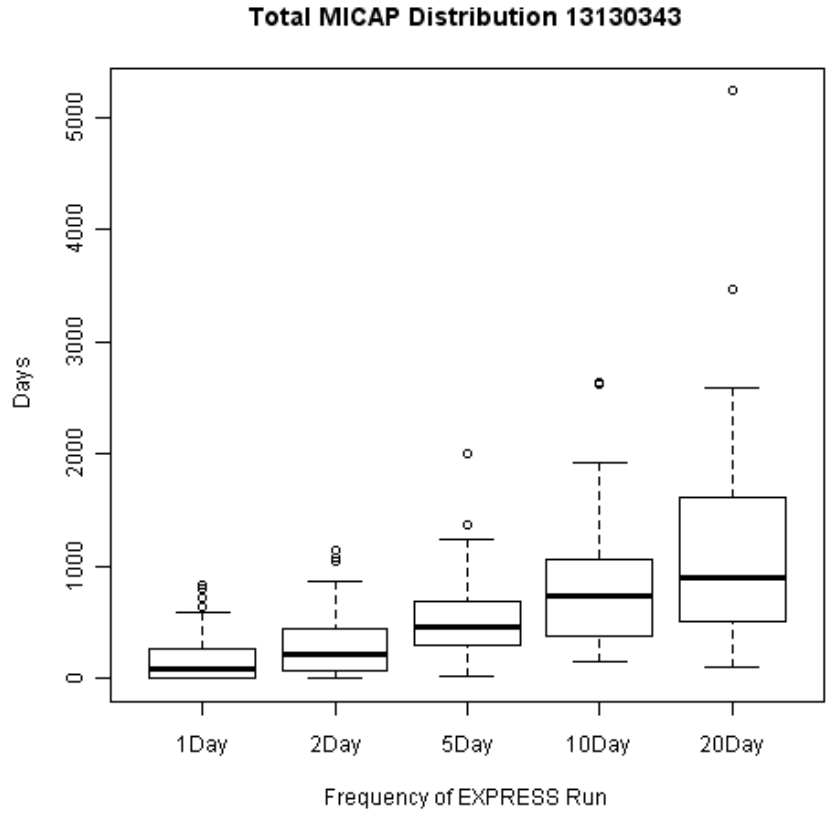

Figure 3: Distribution of total MICAP days: Part 1. 
Williams, Miller, Mattioda

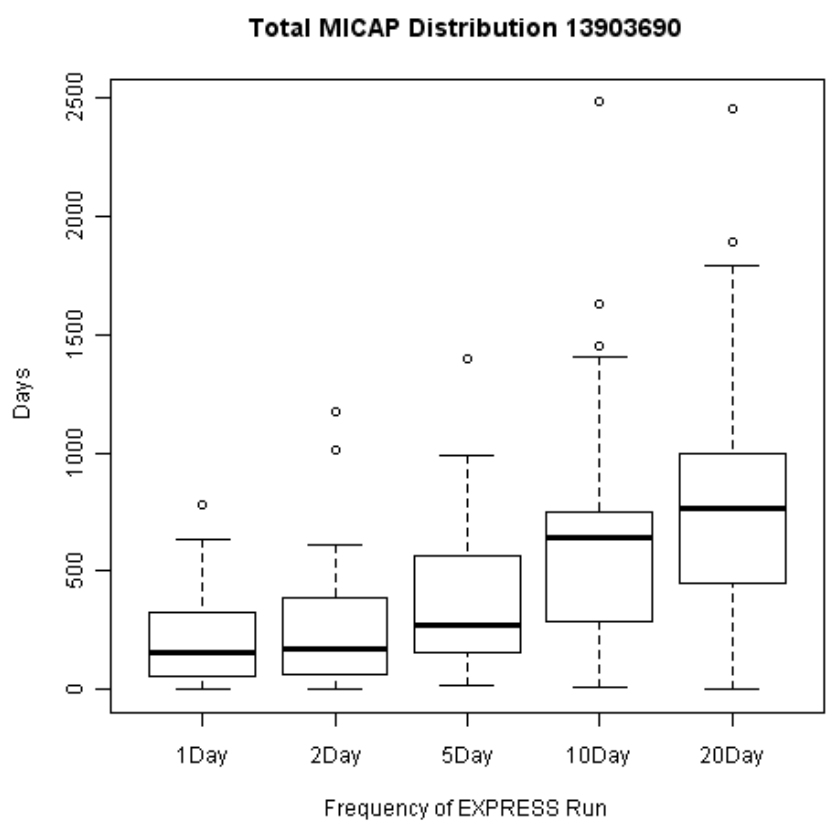

Figure 4: Distribution of total MICAP days: Part 2.

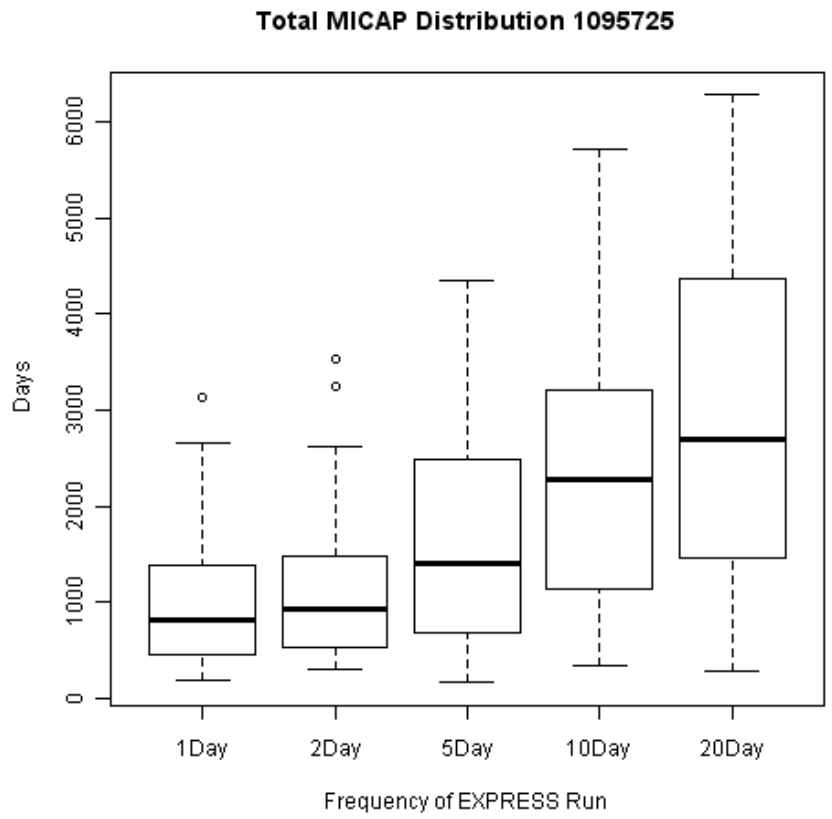

Figure 5: Distribution of total MICAP days: Part 3.

The other important system behavior that is tied to run frequency is repair workload and distribution. In actual operations it is arguable that shop behavior would compensate for some of the reduction in responsiveness with gains in efficiency due to scheduling. For this effort shop capacity is left constant across runs in order to focus analysis on the raw change due to the structure of EXPRESS. Figure 6 shows how shop workload was distributed for the different run periodicities. 

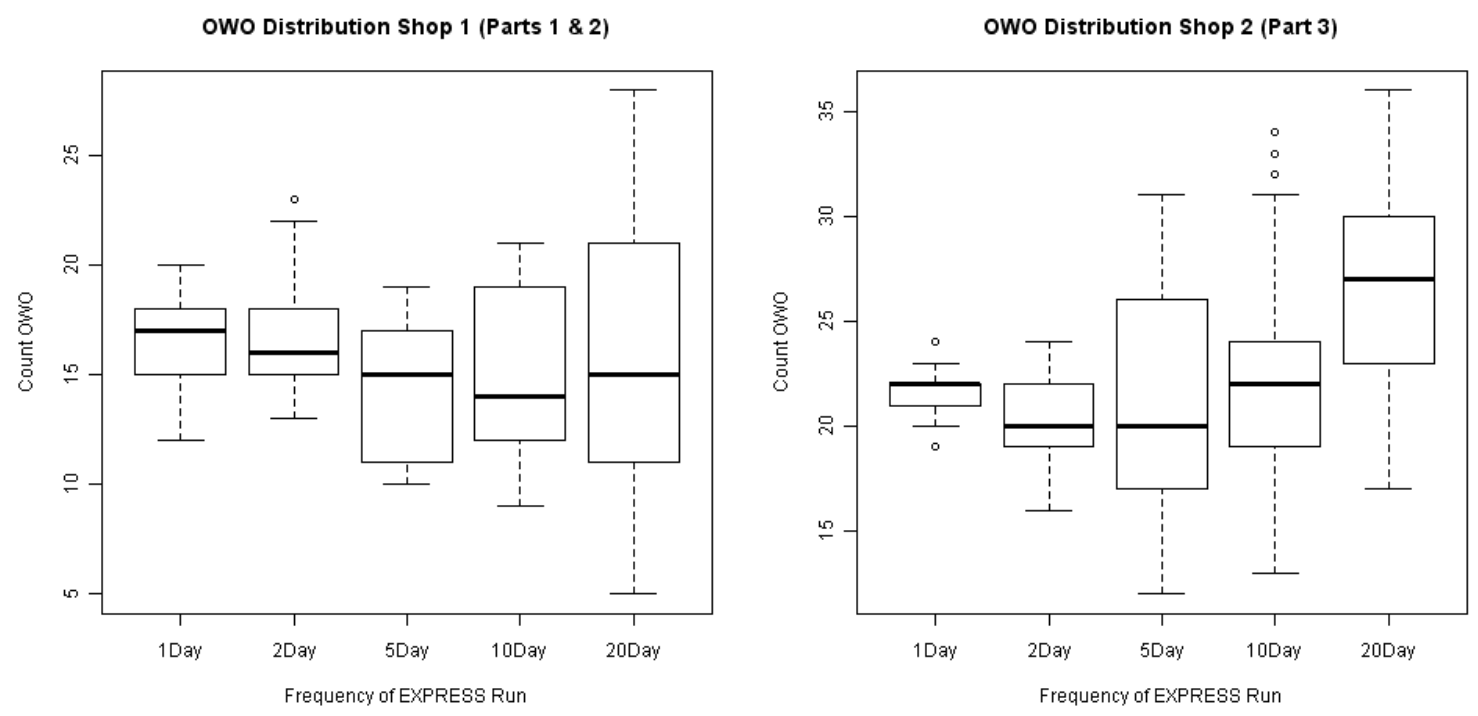

Figure 6: Distribution of OWO by repair shop.

Clearly, across both shops, the variance on workload increases with the number of days between runs. This would be undesirable to a maintenance planner attempting to keep consistent workloads in order to maintain a trained and efficient workforce. Additionally, drastic swings in workload distribution across the parts repaired by a shop could result in the need to retrain and redistribute employees or equipment within the shop. Figure 7 shows the distribution of the portion of labor in shop 1 used to repair part 1 over the different configurations (calculated by OWO_Part1/(OWO_Part1+OWO_Part2) since these are the only two parts modeled for this shop).

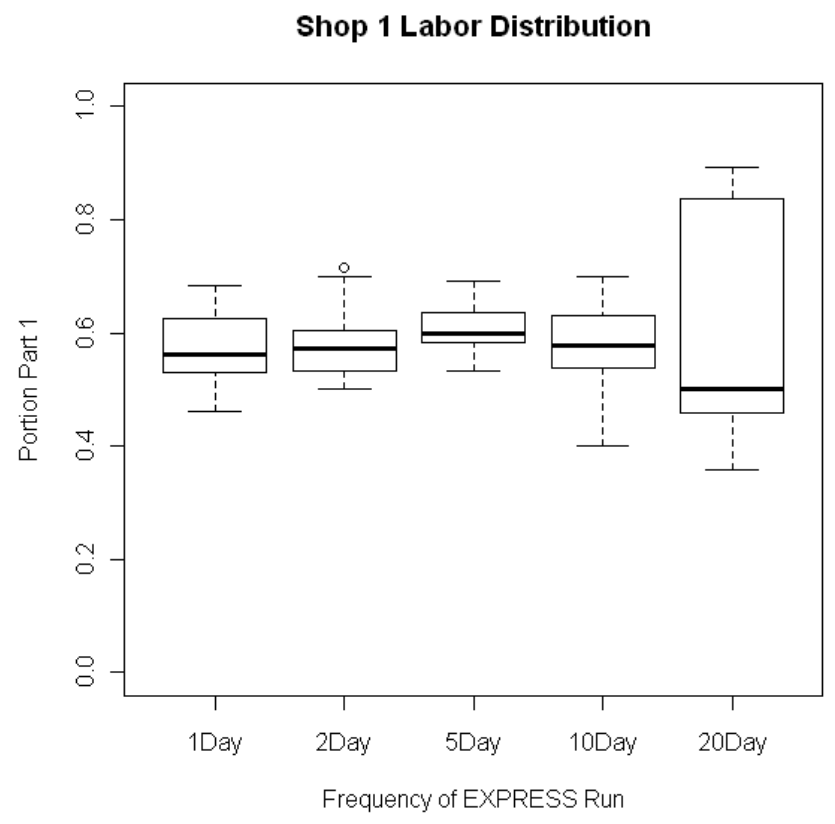

Figure 7: Portion of shop 1 used for part 1. 
The general trend here is for the range of this portion to increase as run frequency decreases. There does appear to be an unexpected benefit found by running weekly as the range is less for this configuration, which may be an interesting focal point of future study. But the increase in range points towards a trend undesirable to the maintenance community.

\section{CONCLUSIONS}

The model used here captures adequate database behavior to produce interesting insights into the question of how often EXPRESS should be run. Output data points to an increasing trend in total MICAP days over the modeled six month period as run frequency is decreased. In general this trend is highly undesirable to the AF under its DREP goals of maximizing responsiveness, however the amount of change is neither quantified nor mapped to any decision criteria in this study. Similarly, there is statistical evidence that repair shop behavior is also negatively impacted, with shop workload becoming more volatile (both overall and between parts repaired within the shop) as the amount of time between EXPRESS runs increases. Given the assumptions used in data collection and model creation for this effort, the statistical evidence indicates that running EXPRESS less frequently negatively impacts the depot repair process's effectiveness, both for the maintainers and the supply chain managers. The interpretation of these impacts, along with how they influence actual system configuration decisions, is left to the EXPRESS user community.

\section{ACKNOWLEDGMENTS}

The views expressed in this article are those of the authors and do not reflect the official policy of the United States Air Force, Department of Defense, or the US Government.

\section{REFERENCES}

Abell, J., L. Miller, C. Neumann, and J. Payne. 1992. "DRIVE: Ehancing the Responsiveness of Depot Repair." Technical Report R-3888-AF, RAND Corporation.

Air Force Material Command. 1995. "Air Force Material Command Studies and Analyses Office Annual Report.” Air Force Material Command. http://handle.dtic.mil/100.2/ADA305838.

Air Force Material Command, AFMC/A9A. 2012. "Interviews with AFMC/A9A Operations Analysts." Interview notes.

Air Force Material Command. 2001. “AFMCI 21-129: Depot Maintenance Management, Depot Repair Enhancement Process (DREP)." Air Force Material Command.

Air Force Material Command. 2004. "EXPRESS System/Subsystem Design Description (SSDD)." Technical Report Contract Number: GS-35F-4381G Task Order Number: FA8770-04-F-0028 CDRL Number: A016, Material Systems Group/SLW, Wright-Patterson AFB, OH.

Air Force Material Command, $428^{\text {th }}$ Supply Chain Management Squadron, 2011. "Interviews with Okla homa City ALC EXPRESS Operators." Interview notes.

Crawford, G.B. 1988. "Variability in the Demands for Aircraft Spare Parts: Its Magnitude and Implications." Technical Report R-3318-AF, RAND Corporation.

Cohen, I., J. Abell, J., and F. Lippiatt. 1991. "Coupling Logistics to Operations to Meet Uncertainity and the Threat." Technical Report R-3979-AF, RAND Corporation.

Department of Defense. 2000. "DODD 4140.61: Customer Wait Time and Definite Delivery." Department of Defense.

Miller, L., and J. Abell. 1992. "DRIVE: Design and Operation of the Ogden Prototype." Technical Report R-4158-AF. RAND Corporation. 
Moore, R., and B. McCormick. 1992. “DRIVE Model Logic.” Working Paper 92-003, AFMC/XPS, Air Force Material Command.

Stafford, T., 2002. "SCOPE Simulation Study of Periodicity in EXPRESS," Powerpoint presentation. AFMC//XPS, Air Force Material Command.

Walker, R., 2012. "Interviews with Robert Walker, Deputy Program Manager of EXPRESS.” Interview notes.

Williams, D., 2012. "Examining EXPRESS with Simulation.” MS Thesis, AFIT/OR-MS/ENS/12-27, Graduate School of Engineering and Management, Air Force Institute of Technology, WrightPatterson AFB, $\mathrm{OH}$.

\section{AUTHOR BIOGRAPHIES}

DAVID WILLIAMS received his B.S. degree from Oklahoma Baptist University in 2007 and M.S. degree in Operations Research from the Air Force Institute of Technology in 2012. He currently serves as a Captain in the US Air Force stationed at Los Angeles AFB, CA, where he is an operations research analyst at the Space and Missile Systems Center. His research interests include combat modeling, computer simulation, and supply chain management. He can be reached at david.williams.98@us.af.mil.

J.O. MILLER is a 1980 graduate of the U.S. Air Force Academy (USAFA) and retired from the Air Force as a Lt. Colonel in January 2003. In addition to his undergraduate degree from USAFA, he received an MBA from the University of Missouri at Columbia in 1983, his M.S. in Operations Research from the Air Force Institute of Technology (AFIT) in 1987, and his Ph.D. in Industrial Engineering from The Ohio State University in 1997. Dr. Miller is Director of the Center for Operational Analysis and an associate professor in the Department of Operational Sciences at AFIT. His research interests include combat modeling, computer simulation, and ranking and selection. He can be reached at john.miller@afit.edu.

DAN MATTIODA received his B.S. degree from Embry-Riddle Aeronautical University in 1997, M.S. in Logistics from the Air Force Institute of Technology in 2002, and a Ph.D. in Business Administration (Marketing and Supply Chain Management focus) from The University of Oklahoma in 2007. He currently serves as a Lieutenant Colonel in the US Air Force stationed at Wright-Patterson AFB, OH, where he is an Assistant Professor and Deputy Department Head, Department of Operational Sciences at the Air Force Institute of Technology. His research interests lie in the areas of production operations, trust and collaboration, and simulation to model supply chain processes. Dr. Mattioda can be reached at daniel.mattioda@afit.edu. 